\title{
INTRODUZIONE AL TEMA MONOGRAFICO
}

\author{
di Patrizia Messina*
}

La crisi di sostenibilità del welfare state sta accompagnando da tempo il declino del modello fordista di sviluppo e di regolazione, facendo emergere la rilevanza di due dimensioni cruciali per lo sviluppo, strettamente interconnesse: da un lato, la dimensione della coesione sociale come bene relazionale in grado di garantire capitale sociale e, quindi, sviluppo di un territorio; dall'atro, la dimensione territoriale stessa entro cui si definiscono le dinamiche del nuovo modo di sviluppo e si genera la coesione sociale.

Le ricerche sul tema consentono di mettere in luce, insieme alla crisi, anche importanti trasformazioni dei sistemi di welfare nei diversi Paesi e Regioni occidentali, sottolineando come, pur con importanti differenze contestuali (Ferrera, 1993; Bertin, 2012), che possono essere ricondotte a diversi modelli di capitalismo (Regini, 2000; Burroni, 2019), la tendenza che sembra accomunare questo "secondo welfare" consista in un riposizionamento dell'attore pubblico nelle reti di governance, con una maggiore integrazione tra pubblico e privato, profit e non-profit, e un maggiore coinvolgimento della società civile.

La prospettiva più interessante delle diverse combinazioni di "welfare mix" che si sta profilando sembra essere quella della "sussidiarietà circolare" (Zamagni, 2013), in cui i cittadini sono ora coinvolti nel processo di pianificazione e di co-produzione dei servizi, superando così la dicotomia pubblico-privato (Stato-Mercato) e aggiungendo la dimensione dalla società civile (Zamagni, Venturi, Rago, 2018). Seguendo questo trend, le trasformazioni più interessanti riscontrabili nelle politiche di welfare possono essere allora sinteticamente descritte come un passaggio da un modello di welfare state (gerarchico, paternalista e assistenziale) ad uno

* Dipartimento di Scienze Politiche, Giuridiche e Studi Internazionali, Università degli Studi di Padova.

ECONOMIA E SOCIETÀ REGIONALE - ISSN 1827-2479 - XXXVII(2) 2019 - INNOVAZIONE SOCIALE E 
di welfare society, o welfare community ("a rete"), che porta con sé un più ampio processo di trasformazione delle relazioni tra Stato, Mercato e Comunità e, quindi, del modo di regolazione dello sviluppo ${ }^{1}$ che caratterizza un dato territorio. Si tratta insomma di una trasformazione epocale, che è stata mirabilmente descritta da Ulrich Beck (2016) come "metamorfosi del mondo".

L'innovazione dei sistemi di welfare sta facendo emergere, negli ultimi anni, insieme alla rilevanza della dimensione territoriale dello sviluppo, anche la necessità/opportunità di coniugare lo sviluppo territoriale con gli obiettivi dello sviluppo sostenibile (Agenda 2030), articolati nelle sue cinque componenti costitutive: ambientale, economica, sociale, politica e istituzionale, tutte strettamente interrelate. Tali dimensioni, per poter essere adeguatamente implementate e analizzate, richiedono tuttavia l'adozione di un approccio integrato ed eco-sistemico. In questa nuova prospettiva, anche le politiche sociali vanno reinterpretate, superando l'approccio settoriale che le ha caratterizzate nel contesto fordista.

Le politiche sociali, volte a produrre servizi alla persona, concepiti ora come "beni collettivi per lo sviluppo", incidono infatti direttamente sia sulla capacità di generare coesione sociale sia sulla qualità della vita di un dato contesto, contribuendo in modo importante a rendere attrattivo (e competitivo) un dato territorio. Questa prospettiva integrata cambia radicalmente il modo di intendere le politiche sociali: da costo improduttivo (come avveniva nel contesto del welfare state), a investimento, soprattutto nei momenti di crisi, in cui diventa indispensabile attivare politiche volte a generare coesione sociale, contrastando la frammentazione e le derive individualiste e conflittuali, tipiche delle fasi congiunturali negative.

D'altro canto, questa metamorfosi richiede, inevitabilmente, un cambiamento anche di modelli organizzativi e culturali obsoleti, a cominciare da quelli della Pubblica amministrazione, generalmente strutturati ancora per settori di policy, che richiedono investimenti mirati e una chiara volontà politica nel perseguire gli obiettivi del cambiamento nel lungo periodo.

1 L'approccio del modo di regolazione dello sviluppo focalizza l'attenzione sulle peculiari dinamiche di regolazione che caratterizzano un dato contesto territoriale e il modo di sviluppo ad esso correlato, analizzando l'interazione delle tre forme idealtipiche della regolazione: economica (Mercato), sociale (Comunità) e politica (Stato). Le caratteristiche di un modo di regolazione sono rilevabili attraverso l'analisi di stili di azione, prassi amministrative e sistema di valori consolidati nell'universo simbolico che accomuna i diversi attori dello sviluppo di un dato territorio. Sussiste quindi una relazione biunivoca tra un dato modo di regolazione e un dato modo di sviluppo. A questo riguardo mi permetto di rimandare a Messina (2012a). 
La medesima trasformazione riguarda però anche le organizzazioni di rappresentanza degli interessi di categoria e delle Parti sociali, chiamate a superare una concezione di difesa degli interessi in senso corporativo, per abbracciare una prospettiva territoriale di sviluppo sostenibile.

Anche le imprese sono coinvolte in questa trasformazione, sia perché i principi della responsabilità sociale di impresa e di territorio vengono da queste sempre più ricercati ${ }^{2}$, sia perché, per le imprese più innovative, si sta assistendo a un processo di ibridazione intersettoriale di particolare interesse in cui, come nel caso delle imprese B-Corp e delle Società Benefit, profit e non profit mostrano di poter coesistere armoniosamente e di poter generare innovazione sociale.

Il medesimo processo di "metamorfosi del mondo", può essere analizzato, insomma, focalizzando l'attenzione sul fenomeno più ampio dell'innovazione sociale che ha sistematicamente segnato l'evoluzione e le trasformazioni dei sistemi sociali, soprattutto nei momenti di crisi epocali come quella che stiamo attraversando, sotto i profili socioeconomico, politico e tecnologico. Molte sono infatti le sfide che mettono in discussione la tradizionale e consolidata idea di sviluppo e il relativo modo di regolazione, che richiedono ora trasformazioni a tutti i livelli.

A questo riguardo, il Centro di Ricerche Internazionali sull'Innovazione Sociale (CeRIIS) della Luiss definisce l'innovazione sociale come:

«Un insieme di azioni che danno vita a "innovazioni relazionali" per una nuova e più efficace interazione tra settore pubblico, grandi imprese e società civile in modo da essere capaci di offrire nuove e concrete risposte a bisogni sociali emergenti» (Flora, 2018: 13).

Si tratta, insomma, di un cambiamento del modo di regolazione che parte da nuove forme di interazioni tra Stato, Mercato e Comunità, a partire dalle innovazioni prodotte nella sfera sociale, anche ad opera di nuove imprese o da imprese leader (Caroli, 2018). Una forma di innovazione generativa di valore, legata alla capacità di trovare nuove soluzioni a bisogni presenti sul territorio, che non vengono soddisfatti né attraverso la regolazione economica (mercato) né attraverso la regolazione politica tradizionale (intervento pubblico di tipo top-down), ma piuttosto attraverso un'azione di governance partecipata in grado di generare comunità e coesione sociale.

2 La letteratura sul tema dalla Corporate Social Responsibility (Csr) è molto vasta; rilevante è soprattutto la trasformazione che il concetto ha avuto negli ultimi anni, entrando in sinergia con i temi dello sviluppo sostenibile e dell'innovazione sociale. Per un approfondimento su questi aspetti cfr. Messina (2019). 
L'innovazione sociale non va confusa con l'impresa sociale, né con le nuove imprese ibride. Essa attiene piuttosto a nuove modalità di decisione e di azione per soddisfare specifici bisogni di una comunità. L'accento va messo, insomma, sul diverso modo di affrontare problemi complessi, che non possono essere efficacemente affrontati e risolti con le modalità organizzative tradizionali, tipicamente gerarchiche, ma richiedono piuttosto un cambiamento organizzativo di tipo reticolare, adottando l'intera gamma degli strumenti a disposizione, dall'uso innovativo delle nuove tecnologie alla formazione delle risorse umane ${ }^{3}$, fino a riguardare forme di coordinamento e collaborazione orizzontali, piuttosto che di controllo verticale.

Questo tipo di innovazione si connota, quindi, anche per una sua componente tecnica e organizzativa, la quale incrementa le capacità di azione della collettività che si mobilita, crea nuovi ruoli, nuove professionalità e nuove relazioni tra gli attori coinvolti, riuscendo persino a coinvolgere nella produzione risorse e capitale umano altrimenti sottoutilizzati. Essa sta dando luogo a un vero e proprio cambiamento di forma, sia organizzativo sia del lessico, per descrivere le pratiche innovative sperimentate sul campo, come: Co-housing; Social-housing; Co-working; Fab lab; Crowd-funding; B-Corp; Corporate Social Innovation e, nell'ambito specifico del welfare territoriale, le Fondazioni di Comunità ${ }^{4}$, per fare solo qualche esempio. Va sottolineato che questa varietà di nuove modalità di azione è accomunata da un dato importante: si tratta infatti di "organizzazioni ibride", che coniugano profit e no profit, pubblico e privato, ma anche diversi settori produttivi (si pensi, ad esempio, alle fattorie sociali).

I contributi che seguono sono accomunati da queste tematiche con riferimento al caso italiano e, in diversi casi, al contesto del Veneto.

Il contributo di MAURIZIO BUSACCA, NICOLETTA MASIERO introduce il tema del welfare territoriale, con particolare riguardo a due programmi attuati dalla Regione Veneto, Alleanze Territoriali per la Famiglia e Piani di intervento in materia di Politiche Giovanili, evidenziandone le componenti di innovazione sociale e il possibile impatto sull'azione istituzionale.

3 Sulla rilevanza di un adeguamento delle politiche della formazione per accompagnare la trasformazione dei sistemi produttivi locali manifatturieri in sistemi locali dell'innovazione, si veda Messina (2012b).

4 Le Fondazioni di Comunità, in particolare, costituiscono un esempio interessante di ibridazione e di innovazione sociale. Un esempio è dato dalla Fondazione di Comunità della Sinistra Piave, fondata nel 2009 da dieci Comuni dell'ex Ulss 7, dalla stessa Azienda Sanitaria e da Banca della Marca Credito Coop, con l'obiettivo di sensibilizzare i cittadini a un'attività di responsabilità condivisa, sostenendo l'azione dei Comuni (soprattutto piccoli) nell'ambito delle politiche sociali del territorio. Cfr.: <http://www.fondazionesinistrapiave.it/>. 
Il contributo di BARBARA BELlotTo, MARTA NALIN, Silvia RANZATO descrive invece le modalità di implementazione del progetto Alleanze Territoriali per la famiglia nel caso del Comune di Padova, mettendo in luce non solo le forme di innovazione sociale ma, soprattutto, il notevole sforzo di innovazione organizzativa e della governance territoriale attuato dall'Amministrazione comunale a partire da un modo nuovo e integrato di concepire e attuare le politiche sociali nella città.

I tre contributi successivi focalizzano l'attenzione sulle novità emergenti nell'ambito del welfare aziendale: CHIARA PATTARO, MARTINA VISENTIN, ANNA ZANNONI presentano un progetto di welfare aziendale, realizzato in Veneto, utilizzando gli strumenti del servizio sociale professionale in una prospettiva di dialogo, confronto e collaborazione tra istituzioni di welfare locale, imprenditori e lavoratori.

David Rossi, Fabio Streliotto, SARA TAglietti, SABrina TOFFOLI, presentano un programma costruito ad hoc, che si propone di integrare diverse forme di welfare (aziendale, contrattuale/bilaterale e pubblico) per generare valore condiviso e coesione sociale.

GIULIO MATTIAZZI focalizza l'attenzione sull'innovazione sociale e il valore aggiunto generati dal coinvolgimento attivo dei lavoratori alle scelte dell'impresa.

Gli ultimi due contributi sono dedicati invece a un'analisi delle imprese B-Corp e Società Benefit in Italia (VALENTINA DE MARCHI, ElEONORA DI MARIA, AMBRA GALEAZZO) e in Veneto (BLERINA BRAMI) mettendo in luce alcune caratteristiche peculiari e l'apporto che questo nuovo tipo di imprese può dare alla sostenibilità dello sviluppo in termini di innovazione sociale responsabile.

Conclude il percorso di approfondimento l'INTERVISTA di ALBERTO MATTEI a TIZIANA BASSO, in quanto componente della Segreteria Cgil Regionale e titolare, fra le altre, delle deleghe alle Politiche industriali e contrattuali del settore privato e alla bilateralità, una testimone privilegiata della contrattazione sindacale in materia di welfare aziendale, che consente di mettere in luce la rilevanza della contrattazione sindacale nel definire le nuove forme di welfare aziendale o come bene di club, riservato cioè solo ai dipendenti dell'azienda e non al territorio in cui essa opera, oppure come bene collettivo per lo sviluppo territoriale, che può assumere la forma di bene pubblico, quando l'attore politico regolatore interviene del processo costituente attraverso apposite forme di regolazione, oppure può assumere la forma di bene comune quando il processo costituente è affidato all'autoregolazione comunitaria tra le parti, anche pubbliche, ma di tipo non gerarchico. 
Queste strategie, volte ad incrementare la dotazione di beni relazionali territoriali, anche attraverso l'attivazione di pratiche partecipative, andrebbero adeguatamente valorizzate nelle politiche pubbliche per lo sviluppo locale sostenibile, soprattutto nei sistemi produttivi locali di piccola impresa.

\section{Riferimenti bibliografici}

Beck U. (2016). The Metamorphosis of the World. Cambridge: Polity Press.

Bertin G. (2012). Welfare regionale in Italia. Venezia: Edizioni Ca' Foscari.

Burroni L. (2019). Capitalismi a confronto. Istituzioni e regolazione dell'economia nei paesi europei. Bologna: il Mulino.

Caroli M.G., a cura di (2018). L'innovazione delle imprese leader per creare valore sociale. Terzo rapporto CeRIIS sull'innovazione sociale. Milano: FrancoAngeli.

Ferrera M. (1993). Modelli di solidarietà. Politica e riforme sociali nelle democrazie. Bologna: il Mulino.

Flora F. (2018). Prefazione a Caroli M.G., a cura di. L'innovazione delle imprese leader per creare valore sociale. Terzo rapporto CeRIIS sull'innovazione sociale. Milano: FrancoAngeli.

Messina P. (2012a). Modi di regolazione dello sviluppo locale. Una comparazione per contesti di Veneto ed Emilia-Romagna. Padova: Padova University Press.

Messina P., a cura di (2012b). Formare per tras-formare. La formazione continua come bene comune per lo sviluppo locale. Padova: Padova University Press.

Messina P., a cura di (2019). Oltre la responsabilità sociale di impresa. Territori generativi e responsabili. Padova: Padova University Press.

Regini M. (2000). Modelli di capitalismo. Le risposte europee alla sfida della Globalizzazione. Bari-Roma: Laterza.

Zamagni S. (2013). Impresa responsabile e mercato civile. Bologna: il Mulino.

Zamagni S., Venturi P., Rago S. (2018). Valutare l'impatto sociale. La questione della misurazione nelle imprese sociali. Impresa Sociale, 11. 